\title{
SISTEMATIZACIÓN DE ESFUERZOS DE INTERNACIONALIZACIÓN EN LA ESCUELA DE CIENCIAS DE LA COMUNICACIÓN COLECTIVA, DURANTE EL PERÍODO 2011-2014
}

\section{SYSTEMATIZATION OF INTERNATIONAL EFFORTS ON THE COMMUNICATION SCHOOL, IN THE PERIOD 2011-2014}

\author{
Harold Hütt Herrera
}

Fecha de recepción: 17 de mayo de 2017 - Fecha de aceptación: 3 de agosto de 2017

\begin{abstract}
Resumen
Este artículo tiene como objetivo presentar un resumen de los resultados obtenidos por la Escuela de Ciencias de la Comunicación Colectiva (ECCC) en materia de internacionalización, durante el período 2011-2014. Asimismo, detalla las diversas metodologías utilizadas en este proceso, para efectos de compartir las lecciones aprendidas y aportes pedagógicos de los docentes que estuvieron involucrados en este tipo de estrategias. De manera complementaria, ofrece un soporte bibliográfico, que permite sustentar las actividades ejecutadas, con elementos que se han venido teorizando en esta materia durante los últimos años. Además de sistematizar los elementos incorporados en estas actividades, para lo cual se realizó un análisis documental y entrevistas, se hacen algunas reflexiones y recomendaciones para impulsar el abordaje conceptual y metodológico, siendo que este tipo de prácticas son de gran valía tanto para docentes como para estudiantes, enriqueciendo los procesos de análisis, así como de intercambio de planteamientos y perspectivas de contexto. Asimismo, y después de hacer una detallada descripción de los proyectos implementados y las lecciones aprendidas, se concluye que las actividades de internacionalización requieren del compromiso e involucramiento de las partes, donde la planificación adquiere una relevancia mayor, en aras de garantizar el éxito de las actividades, y que en general, las nuevas tecnologías ofrecen múltiples canales y herramientas que facilitan el desarrollo de metodologías creativas e innovadoras, que estimulen la participación activa del estudiante, y permitan potenciar el alcance de estas interacciones académicas y multiculturales.
\end{abstract}

Palabras clave: Internacionalización, Sistematización, Proyectos colaborativos, Videoconferencias, Estrategias didácticas.

\begin{abstract}
This paper has the objective of introducing a resume of the results obtained through the Communication School, regarding the internationalization experiences implemented during the period 2011-2014. Also, includes a detailed description of the methodologies used on this internationalization process, with the aim to share the learned lessons and pedagogical contributions of the professors who were involved on this kind of strategies. In complement of this content, the article make a relation with the content with some main concepts about some authors have been writing during last years. In addition of the systematization of the international activities, the article offer some reflections and suggestions to promote the conceptual and methodological aspects on this internationalization practices, recognizing that these kind of practices have a great value for professors and students, strengthening the analysis process, knowledge exchange and context perspectives. In addition, and after to make a
\end{abstract}

1 Profesor Asociado y Coordinador de la Estrategia de Internacionalización, Escuela de Ciencias de la Comunicación Colectiva, Sede Rodrigo Facio, Universidad de Costa Rica, qmcostarica@racsa.co.cr 
detailed description of the implement projects and the learned lessons, based on documents analysis and interviews, the author conclude that the international activities required the commitment of the professors which develop this kind of projects, and the planning acquire a relevant condition to succeed on this purpose. Also, it is necessary to take into account that new technologies offering multiples channels and tools that facilitate the implementation of creative methodologies that promote an active student participation and to strengthen the impact of this academic and multicultural experiences.

Keywords: Internationalization, Systematization, Collaborative projects, Videoconferences, Didactic strategies.

\section{Presentación}

A partir del trabajo realizado en la Escuela de Ciencias de la Comunicación Colectiva, en el marco de la Estrategia de Internacionalización -durante el período 2010-2014-, se ha desarrollado la presente sistematización de esfuerzos, tomando como referencia las principales actividades implementadas con contrapartes internacionales, en procura de describir las metodologías y tecnologías que se utilizaron, y principalmente, de rescatar las lecciones aprendidas y sugerencias que puedan servir de base a docentes y/o estudiantes, que deseen implementar este tipo de iniciativas. Asimismo, el artículo presenta una serie de conclusiones generales, que de igual manera, sirven como base de sustento para el diseño y planificación de este tipo de proyectos.

A manera de contexto general, es oportuno mencionar que la internacionalización de la educación superior ha sido una tendencia mundial en las últimas décadas, incrementando significativamente su impacto, a partir del desarrollo de las nuevas tecnologías de la información y la comunicación (TIC). En este sentido, "la sociedad del conocimiento está planteando la creación de una nueva modalidad educativa, que tiene escalas diferenciadas, que no tiene fronteras, que permite un fraccionamiento de los procesos educativos localizando en un país las tutorías, en otro los softwares o hardwares, en otros los alumnos o los profesores, en una lógica que permite una educación supranacional”. (Rama, 2007 p. 34)

Lo anterior contribuye a una interacción permanente entre diversas culturas, contextos e instituciones educativas, lo que genera un entorno propicio para el aprendizaje global, facilitando esto que el estudiante comprenda con mayor claridad el alcance y las implicaciones de las acciones globales, desde diversas perspectivas y condiciones.

Esto a facultado lo que Rama (2007) denomina el surgimiento de los "modelos híbridos de educación" (p.35) combinando clases virtuales y presenciales, lo que a su vez facilita el impulso de aprendizajes en los contextos local e internacional, a partir de estas nuevas posibilidades que surgen para el intercambio de conocimientos e información.

La virtualización que ofrecen las TIC expande las posibilidades de interacción y democratiza, en gran medida, el acceso a esta interacción global, implicando desde luego, la adopción de nuevas y más creativas metodologías de aprendizaje y el impulso de nuevos idiomas, tanto en la población docente como estudiantil. En referencia a este elemento, se estima que "para el año 2025, cuando el mundo alcance los 160 millones de estudiantes universitarios, casi 40 por ciento de ellos habrán participado en alguna experiencia de educación híbrida, virtual, no presencial o a distancia" (Rama, 2007, p.36).

Por su parte, según indica Didou (2014) la UNESCO ha venido monitoreando y analizando el avance de la internacionalización de la educación superior en el mundo, con 70 titulares que participan en las Cátedras de Educación, concluyendo en sus acuerdos que es necesario "emprender sistemáticamente ejercicios comparativos de seguimiento de los programas de internacionalización" (Didou, 2014, p.3) debido a la importancia que reviste este tema. Esta relevancia se basa, según detalle Didou (2014), en los siguientes aspectos:

a. Mercado y comercialización de los sistemas educativos, con todas las potencialidades que implica la virtualidad.

b. Movilidad de los profesionales en las zonas de integración económica. 
c. Desplazamiento de científicos.

d. Co-grados y programas educativos compartidos (entre dos o más países).

En el marco de este análisis realizado por los miembros de la cátedra, se abordó también la relevancia de las TIC, y el uso de las nuevas modalidades de aprendizaje en línea (Didou, 2014, p. 4)

En este mismo sentido, se indica que "una de las tendencias dentro de los programas de perfeccionamiento de las instituciones de educación superior (IES) en la actualidad es la internacionalización" (Domínguez, s.f., p.1), lo que es concordante con la inclusión de este elemento dentro de los parámetros de evaluación en los procesos de reacreditación, tal y como lo estipula el Sistema Nacional de Evaluación Superior (SINAES), por ejemplo, en Costa Rica.

Adicionalmente, Domínguez (s.f.) hace hincapié en cómo la generación de conocimiento se ha convertido en un elemento clave para el desarrollo de los países, por lo que la educación, bajo esta perspectiva de interacción global, constituye un pilar fundamental para cumplir este propósito.

Desde esta óptica, Moncada (2011) coincide con que la investigación y la innovación determinan la competitividad de las economías, por lo que el conocimiento es un elemento que debe ser integrado en el aparato productivo. Según afirma: "la cooperación internacional entre universidades es un elemento intrínseco en los procesos de generación de conocimiento científico y del desarrollo humano que apuntan a la innovación" (Moncada, 2011, p. 22).

No obstante, indica que este proceso (la internacionalización) debe ser solidario y debe concebirse como "un bien público y un derecho social, por lo que ha de estructurarse bajo los principios de reciprocidad y respeto a la diversidad" (Moncada, 2011, p. 22).

Adicionalmente, Moncada (2011) destaca el rol que tienen las Universidades en el fomento de una conciencia crítica, y del impulso de una herencia cultural, así como un análisis más profundo sobre el ser humano, su función social y su fin trascendente, de cómo el quehacer universitario debe orientarse a la solución de problemas y a la búsqueda constante del conocimiento.

Según agrega Moncada "la internacionalización implica infundir entre los estudiantes, el personal académico y administrativo, nuevos conocimientos, habilidades y actitudes que les permitan funcionar de manera eficaz en un medio internacional y multicultural" (Moncada, 2011, p. 25). Es claro, en este sentido, que los autores y tendencias son concordantes en la importancia de adoptar prácticas de interacción, que faciliten el intercambio de conocimientos, así como la comprensión de contextos culturales, políticos, económicos y sociales, propios de la diversidad mundial.

A lo anterior, se adiciona que la internacionalización "se presenta como una estrategia complementaria de las tendencias de la globalización, en el sentido de que puede contrarrestar los efectivos negativos y perversos del citado fenómeno... sin olvidar que el origen del mismo es la universalización del conocimiento" (Peña y Jiménez, 2014, p. 150). Asimismo, ambas autoras mencionan que uno de los objetivos de las universidades, es tener la capacidad de realizar lecturas adecuadas del entorno y contribuir proactivamente a generar soluciones para el futuro, el cual a su vez, se verá afectado por la interacción entre países, y por las consecuencias de estas relaciones. (Peña y Jiménez, 2014, p.150).

Sobre este particular, Gacel-Ávila (2006) citados por Peña y Jiménez (2014, p.151), destacan los postulados de la Declaración de París de la UNESCO (1998), donde se establece que la educación superior debe hacer énfasis en los siguientes aspectos de cooperación internacional:

- El fortalecimiento de los instrumentos de cooperación internacional y regional, así como la capacidad nacional en gestión de la cooperación.

- La orientación de la cooperación internacional para la investigación científica y tecnológica debe contribuir a: 1) La instalación en los países con menor desarrollo de capacidades científicas de excelencia; 2) la formación de jóvenes investigadores insertos en sus propias realidades sociales, y 3) el diseño de una agenda de investigación acorde con los valores y prioridades de la región y conforme a una perspectiva mundial. 
- La cooperación horizontal ofrece posibilidades inéditas que permiten intercambiar y complementar capacidades humanas, físicas y financieras de los grupos de investigación, y promover un desarrollo endógeno y homogéneo de esas capacidades.

- Contribuir a comprender, interpretar, preservar, reforzar, fomentar y difundir las culturas nacionales y regionales, internacionales e históricas, en un contexto de pluralismo y diversidad cultural.

- La cooperación internacional tiene que descansar en la solidaridad, el respeto y el apoyo mutuo, y en una asociación que redunde, de modo equitativo, en beneficio de todos los interesados. (Gacel-Ávila, 2006, pp.49-50)

Asimismo, se destaca que "la cooperación universitaria se presenta en una amplia variedad de modalidades y niveles: intercambio académico de profesores y estudiantes, proyectos de investigaciones conjuntas, acuerdos bilaterales, multilaterales o regionales, programas educativos conjuntos, dobles grados, intercambio de expertos, reuniones científicas, cortutelas, entre otros" (Peña y Jiménez, 2014, p. 152).

Sin lugar a dudas, la diversidad de opciones y los niveles de avance de la internacionalización son variables entre universidades y países, pero en general, la conclusión es que esta tarea reviste una actividad de esencial importancia para lograr la inclusión en las discusiones globales que se generan en torno a los nuevos conocimientos y sus aplicaciones, en el marco del desarrollo de las naciones.

\section{Sistematización de experiencias de internacionalización en la Escuela de Ciencias de la Comunicación Colectiva (ECCC) durante el período 2011-2014}

A continuación se detalla la sistematización de los principales proyectos desarrollados en la ECCC durante el período 2011-2014, que han implicado la internacionalización, desde el ámbito académico y/o investigación, según cada caso:

\section{El uso de teleconferencias en el contexto internacional. Caso: ¿cómo utilizar las redes sociales en procesos de investigación?}

Las teleconferencias son un mecanismo práctico y efectivo para promover intercambios académicos, debido a la facilidad con que pueden ser programadas e implementadas. El único requerimiento es contar con una tecnología adecuada, un salón de clase o espacio adecuado, y desde luego, un metodología previamente definida.

En este sentido, es necesario indicar que las metodologías y formatos que ofrece esta modalidad son diversos, y dentro de ellos, se pueden destacar los siguientes: conferencia en vivo o pregrabada, conferencia con espacio para preguntas, conferencia con un comentarista que complemente o amplíe la información y conferencia interactiva, entre otras modalidades.

Los anteriores son algunos ejemplos metodológicos, pero desde luego, la creatividad de los docentes es muy valiosa en este tipo de actividades, ya que permite darle un mayor dinamismo al proceso, ajustándolo a los requerimientos y características del grupo y del tema.

\section{Caso: Videoconferencia sobre las redes sociales como mecanismo de recolección de datos en la investigación}

En el año 2011 se programó la primera teleconferencia internacional, en el marco de la Estrategia de a ECCC, para lo cual se convocó a la Doctora Natalie Undelberg de la Universidad Central de Florida, quien es socióloga, y para entonces, había venido desarrollando diversas experiencias para 
recolectar datos para proyectos de investigación, a través del uso de redes sociales. También se invitó como comentarista a la Doctora Joye Gordon de la Universidad Estatal de Kansas, quien complementó los contenidos abordados durante la sesión.

En esta ocasión, se utilizó la plataforma Skype, con una conexión alámbrica, procurando la comunicación tripartita, tanto en audio, como en video.

A partir de las lecciones aprendidas durante este proceso, se sugieren las siguientes recomendaciones:

a. Coordinar la dinámica de previo con la contraparte, incluyendo los detalles relativos a tiempo, abordaje del tema y cualquier otro elemento que se considere relevante.

b. Probar la tecnología en días previos a la actividad, para corroborar que todo funcione adecuadamente.

c. Realizar la conexión 30 minutos antes del evento, para poder subsanar cualquier detalle que fuera necesario. En este sentido, es importante indicar que herramientas como Skype solicitan actualizaciones con regularidad, y al momento de la videoconferencia, puede ser necesario realizar este procedimiento (se activa de forma automática), el cual toma varios minutos.

\section{Principales retos:}

Metodología idónea: uno de los principales retos en este proceso, es identificar una metodología de trabajo idónea, para que la actividad sea dinámica y participativa, pero principalmente, que permita el desarrollo de aprendizajes significativos a través de un método constructivista.

Tecnología adecuada: la tecnología pareciera ser un factor "dado por defecto", pero en realidad, es importante contar con las condiciones idóneas, según las previsiones que se hayan tomado para efectos de interacción, el tamaño de la audiencia, y desde luego, las expectativa de interacción.

Asistencia: uno de los principales aprendizajes obtenidos en el proceso, es que la asistencia a actividades "libres" no es tan intensa como se había previsto al inicio, por lo que se recomienda que estas actividades puedan programarse en el marco de algún curso específico y, eventualmente, "abrirla al público en general"; y en el caso de la ECCC, anunciarla como una actividad de "horas conferencia", lo que incentivará una mayor participación.

\section{Ficha resumen:}

Proyecto: Teleconferenica

Tema: El uso de las redes sociales en procesos de investigación

Fecha de ejecución: II ciclo, 2011

Docentes involucrados:

- Conferencista: Natalie Undelberg: Central Florida University (CFU)

- Comentarista: Joye Gordon, Kansas State University (KSU)

- Coordinador y moderador: Harold Hütt, Universidad de Costa Rica (UCR)

Total de beneficiarios: 10 estudiantes de la UCR

\section{Un modelo de trabajo colaborativo internacional: el caso de Popchips}

El desarrollo de proyectos colaborativos en la docencia potencia y faculta el trabajo en equipo entre docentes y estudiantes, fomentando el intercambio de experiencias y conocimientos, y aunado a esto, en los proyectos internacionales, permite adicionar un enfoque multicultural. 
Es importante tomar en cuenta que "existen numerosos estudios que resaltan las ventajas del aprendizaje colaborativo, apoyado por ordenador (...) en los que se trata de analizar las posibilidades colaborativas en el proceso de aprendizaje" (Koschmann y Lipoponen citados por Lorraine y Miyata, 2013, p. 2), utilizando entornos virtuales.

En este sentido, Holland \& Muilenburg (citados por Sáez, Lorraine y Miyata, 2013) consideran que el trabajo cooperativo es positivo, incluso desde una perspectiva psicológica, ya que en comparación con el aprendizaje individual y competitivo, el aprendizaje colaborativo mejora considerablemente la autoestima, desarrollando adicionalmente orientaciones y oportunidades para desarrollar interacciones grupales en la que se identifican y resuelven problemas.

Por su parte, Serrano, María de los Ángeles (2010), afirma que existen varios elementos que conforman los proyectos colaborativos:

a. La metodología debe fundamentarse en concepciones educativas acordes con la audiencia.

b. Los objetivos de la actividad deben estar debidamente definidos, y deben estar vinculados a un contexto real, vinculándose a competencias como el pensamiento crítico y la solución de problemas.

c. Debe utilizarse una tecnología adecuada, para facilitar la interacción entre las partes.

Asimismo, y según indica Serrano, María de los Ángeles:

los proyectos colaborativos ofrecen a los estudiantes una forma diferente de aprender en comparación con la enseñanza tradicional. Ofrece cierta libertad para la recolección de materiales en diferentes medios de comunicación, y luego organizarlo para la presentación de las tareas que se sugieren como actividades que conforman los proyectos colaborativos. El ejercicio de seleccionar un tema, diseñar un proyecto, reunir información, organizar material y analizarlo, requiere de gran iniciativa. (2010, p. 6)

\section{El caso de Popchips}

En el segundo ciclo del 2011, y en coordinación con la profesora Linda Lee de la Universidad de Kansas - desde el curso Messages Development-, el profesor Harold Hütt de la Escuela de Ciencias de la Comunicación Colectiva (ECCC), UCR, -desde el curso Relaciones con los Medios de Difusión-, trabajaron de manera conjunta en el diseño de un proyecto colaborativo, para la empresa Popchips, ubicada en San Francisco, California.

La empresa Popchips se encarga de la fabricación de papas fritas, bajo una nueva modalidad de cocimiento, que permite combinar las características de las palomitas de maíz, con las papas tostadas regulares, permitiendo obtener una reducción en las grasas saturas que se retienen en el producto. Ketih Belling, Co-fundador y Director de la empresa, afirma que: "Popchips es más que un snack, este es un nuevo giro de negocio en este mercado” (recuperado de www.popchips.com).

El reto de comunicación era cómo diseñar mensajes que permitieran sustentar una estrategia de comunicación, y que pudiera funcionar en ambos países (Estados Unidos y Costa Rica). A partir de lo anterior, se definió una estrategia metodológica que consistía en:

a. Una introducción al proyecto por parte de los profesores involucrados, con el fin de brindar las instrucciones a los estudiantes.

b. El Vicepresidente de la Compañía facilitó la grabación de un video, con su directora de mercadeo, para brindar más insights al grupo.

c. Se compartieron los lineamientos promocionales de la empresa, donde se incluía la reciente -en ese entonces-, contratación del actor Ashton Kutcher. 
Durante este proceso, los estudiantes desarrollaron estrategias para el lanzamiento del producto, incluyendo desde luego, propuestas para la introducción del mismo en el mercado costarricense: el proceso estimuló a ambos grupos a crear spots para radio, estrategias "on-line", comunicados de prensa, y principalmente, trabajar en la esencia de los mensajes claves.

\section{Recomendaciones}

a. Motivación de los estudiantes: al inicio del proceso, deben realizarse acciones para motivar a los estudiantes a participar de la iniciativa, y asumir con responsabilidad y entusiasmo el proyecto.

b. Confección de una estrategia metodológica dinámica: la metodología elegida debe resultar retadora, pero también participativa y gratificante para los involucrados. En este sentido, es importante trabajar con los grupos involucrados, para incentivar el sentido de pertenencia y afiliación hacia el proyecto, por parte de todos los integrantes, y motivarlos para incentivar el trabajo colaborativo, rescatando las ventajas de la multiculturalidad.

c. Coordinación del proceso de implementación: Durante el diseño, implementación y evaluación, los docentes deben estar activamente involucrados, en el marco de una coordinación constante, para lograr un proceso exitoso.

d. Evaluación de los resultados del proceso: Siempre es importante procurar una evaluación y cuantificación de los resultados obtenidos, para que tanto docentes como estudiantes, puedan percibir el aprendizaje y el progreso alcanzado.

e. Documentación: es muy recomendable tener la oportunidad de archivar documentos, producciones y fotografías, que puedan irse generando durante todo el proceso, así como el registro de estudiantes respectivo.

Ficha resumen:

Proyecto: Popchips

Tema: Desarrollo de mensajes claves para estrategia comercial

Fecha de ejecución: II ciclo, 2011

Docentes involucrados:

- Kansas University: Linda Lee

- Universidad de Costa Rica: Harold Hütt

Total de beneficiarios: 25 estudiantes (12 estudiantes de la UCR y 13 de KU)

\section{Software colaborativo para la docencia: el caso del Voicing Board}

El uso de las nuevas tecnologías de la información y la comunicación han potenciado las posibilidades y alternativas para el desarrollo de proyectos colaborativos, y en el caso de la académica, esto ha tenido incidencias positivas en la investigación, en la acción social y en la docencia.

Es por esta razón, que el software se ha constituido como un pilar fundamental en el proceso de internacionalización, dado que se convierte en el medio y/o canal, para promover la interactividad entre grupos, estudiantes y/o docentes, indistintamente de cuál sea su localización geográfica.

Se hace referencia al hecho de que "para que el crecimiento y enriquecimiento de los procesos de enseñanza puedan darse, es necesario que los docentes tengan capacidad, conocimientos y habilidades para el manejo de tecnologías educativas en el aula y administración de plataformas" (Claudia y Martínez, Evelio, 2008, s.n.). Para que este proceso sea exitoso, el docente debe conocer e identificar las 
TIC más adecuadas a sus necesidades y expectativas, en términos del proyecto o actividad a desarrollar, tomando en cuenta desde luego, que el mismo pueda ser utilizado por la contraparte.

Un elemento valioso es que

el uso de las TIC en la educación eleva la calidad del proceso educativo porque permiten superar las barreras de espacio y tiempo donde hay una mayor comunicación e interacción entre profesores y alumnos. Lo anterior lleva a que exista una construcción distribuida de diferentes fuentes de información donde se participa de manera activa en la construcción del conocimiento y así se potencializan los individuos que en este proceso participan, puesto que se desarrollan una serie de habilidades por lo que este contexto implica. (Islas, Claudia y Martínez, Evelio 2008, s.n.).

Dentro de los beneficios que ofrecen las plataformas virtuales, se pueden destacar: el impulso de la conectividad multipartita, tanto sincrónica, como asincrónica, el impulso de la creatividad y la facilitación de un espacio para el aprendizaje colaborativo. No obstante lo anterior, para que el proceso sea exitoso es necesario contar con una estrategia metodológica adecuada, y coordinada entre las y los docentes que estén coordinando y supervisando el proceso de implementación, para garantizar que existan objetivos de aprendizaje específicos, y que el cumplimiento de los mismos pueda ser monitoreado y evaluado.

En cuanto al tipo de herramientas, existen muchas opciones, dentro de las cuales destacan: Blackboard, Moodle, Google Docs, Training Coordinator Tralcom (www.tralcom.com), WebCT.com (www.webct.com), Learning Space, Lotus (www.lotus.com), LearnLinc (www.learnlinc.com), Forum Enterprises, Virtua-U y PB Works, entre otras.

\section{El caso del Voicing Board}

Como una iniciativa del profesor Aarón Mena, se llevó a cabo un proceso de coordinación con la Universidad de Ibaraki, la Universidad Abierta de Japón, la Universidad de Utsunomiya y la Universidad de Soka; todas de Japón, a través de un grupo de investigación en tecnología educativa denominado UMEGUMI.

A partir de este acercamiento se inició un proyecto para desarrollar software colaborativo, que facilitara los procesos de aprendizaje, para lo cual se diseñó el Vocing Board. Este software fue donado por UMEGUMI a la Unidad de METIC'S de la UCR en el año 2014, y puesto a disposición de la comunidad universitaria.

De acuerdo con la información suministrada por el profesor Aarón Mena, quien participó activamente en este proceso, el Voicing Board es una herramienta para el desarrollo del aprendizaje colaborativo, a través de la elaboración de historietas en formato "Manga".

Este software es de utilidad para el abordaje de contenidos específicos, en diversas áreas del conocimiento, impulsando dos competencias de manera transversal: pensamiento polifónico y trabajo colaborativo.

\section{Ficha resumen:}

Proyecto: Software colaborativo

Tema: Vocing Board

Fecha de ejecución: año 2014

Docentes involucrados:

- $\quad$ Universidad de Ibaraki: Hideyuki Suzuki

- Universidad de Soka: Hideo Funaoi

- Universidad Abierta de Japón: Horoshi Kato

- Universidad de Costa Rica: Aaron Mena

Total de beneficiarios: este software cuenta con 500 usuarios registrados entre docentes y estudiantes hasta el presente año (2016). 


\section{El uso de la Técnica Phillips 66, en el análisis de manejo de crisis. El caso de Carnival Cruice}

En el marco de las herramientas grupales, la Técnica Phillips 66 ha tenido un destacado reconocimiento, debido al tipo de abordaje y análisis que permite entre los participantes. De acuerdo con Barbero Alberto (2011), "esta técnica consiste en dividir al grupo en grupos de 6 personas y pedirles que discutan durante 6 minutos sobre un tema que han de llegar a una conclusión y presentarla al grupo". (s.n.)

Esta técnica, según agrega Barbero (2011), se compone de tres etapas principales:

a. El facilitador explica la técnica y brinda las instrucciones generales, divide al grupo en subgrupos, y les indica la forma en que deben proceder (instrucciones generales). Asimismo, formula la pregunta sobre el tema a tratar y se encarga de llevar el control del tiempo.

b. Cada subgrupo elige un coordinador y un secretario. Cada persona expresa sus opiniones y el secretario se encarga de hacer la síntesis respectiva.

c. Se reúne al grupo en una plenaria (o grupo integrado nuevamente), y los secretarios se encargan de transmitir la posición de sus respectivos grupos de trabajo.

Es importante anotar, que aunque esta es una técnica relativamente simple, motiva a la reflexión y participación activa de todos los miembros del equipo, logrando una valiosa síntesis de ideas.

\section{El caso de Carnival Cruise}

El caso que se decidió analizar, para efectos de la implementación de esta dinámica, fue el de la empresa "Carnival Cruise", utilizando como referencia un crucero que se quedó sin energía en el Atlántico, dejando a los pasajeros "atrapados" por más de una semana, con limitaciones de comida, fallas en el sistema eléctrico, y causando múltiples afectaciones a las personas que debían llegar al puerto en una fecha determinada para tomar sus respectivos vuelos de regreso.

Para efectos de esta actividad, se hicieron algunas variaciones en la dinámica, las cuales implicaron lo siguiente (todo se desarrolló en idioma inglés, con el fin de incentivar a los estudiantes de la UCR a practicar este segundo idioma):

a. Se realizaron varias gestiones de coordinación entre los docentes involucrados (Harold Hütt - UCR y Barbara DeSanto - KSU), con el fin de definir el tema, contenido y metodología.

b. Se suministró a los estudiantes un artículo académico publicado sobre este caso de "Administración de Crisis", para que lo estudiaran de previo a la fecha pactada para la actividad.

c. Se realizó la conexión vía Skype para establecer la comunicación, los docentes dieron unas palabras iniciales y explicaron la dinámica respectiva. Posteriormente, la profesora Bárbara DeSanto, quien además era la autora del artículo) hizo una breve introducción.

d. Los grupos de ambas universidades se dividieron en 4 subgrupos (esto fue estimado de acuerdo con la cantidad de estudiantes), y cada subgrupo debía analizar un punto específico de la estrategia: aciertos, desaciertos, acciones que se pudieron haber ejecutado de previo al accidente y acciones que se pudieron haber ejecutado de manera posterior. Esta segregación temática fue la misma para los grupos de ambas universidades.

e. Se nombraron relatores en cada equipo, y posteriormente, los equipos homólogos (subgrupos con el mismo tema asignado) compartieron sus impresiones y criterios, a través de los respectivos relatores.

f. Al final se abrió un espacio para comentarios y preguntas.

g. Los docentes realizaron comentarios finales, a manera de cierre y conclusión.

h. Se dio por finalizada la actividad. 
Una vez que concluyó el proceso, se hizo una valoración general de la actividad con los estudiantes, quienes manifestaron sentirse muy satisfechos con el ejercicio, y destacaron haber podido intercambiar criterios académicos con estudiantes de un contexto cultural distinto.

\author{
Ficha resumen: \\ Proyecto: Técnica Phillips 66 \\ Tema: Administración de Crisis / Carnival Cruise \\ Fecha de ejecución: II ciclo 2013 \\ Docentes involucrados: \\ - Kansas State University: Bárbara DeSanto \\ - Universidad de Costa Rica: Harold Hütt
}

Total de beneficiarios: 52 estudiantes ( 24 estudiantes locales)

\title{
5. Investigación colaborativa para la prevención del Dengue en la Zona Atlántica
}

En el año 2012, la profesora Lissette Marroquín inscribió el proyecto denominado "Fomento a la capacidad para investigaciones basadas en la participación de la comunidad dirigidas hacia campañas de salud pública para la prevención y control de la epidemia de dengue en la provincia de Limón, Costa Rica", cuya implementación se extendió hasta el año 2014. Este proyecto constituyó la primera iniciativa en el marco de la estrategia de internacionalización que permitiría trabajar una investigación en un marco de trabajo internacional, vinculando a docentes y estudiantes de la Universidad de Kansas y la Universidad de Costa Rica.

De esta forma, dio inicio lo que sería un proceso participativo e interdisciplinario, que permitiera identificar las características de la población de la zona, en aras de tener los cimientos adecuados para el lanzamiento de una campaña de comunicación y sensibilización que permitiera mitigar el impacto del zancudo Aedes Aegypti en la zona.

Es necesario anotar que una de las razones por las cuales había una alta incidencia de contagios en esta área, era por cuestiones de carácter cultural, según la información preliminar que se obtuvo del Ministerio de Salud, y que impedían que las personas colaboraran con la eliminación de criaderos potenciales del mosquito.

En este sentido, y de acuerdo con la información suministrada por la docente Lissette Marroquín, se llevó a cabo el proceso de diagnóstico, y posterior implementación de una campaña piloto, para la prevención del Dengue en las Comunidades Luis XV y El Humo (San Antonio)

Durante las primeras fases del trabajo, las actividades que se desarrollaron fueron las siguientes:

a. Mapeo de actores comunitarios: para estos efectos, las investigadoras realizaron entrevistas a funcionarios de instituciones públicas y organizaciones de la localidad, logrando además llevar a cabo un mapeo de líderes locales.

b. Primer Contacto: se realizaron entrevistas con líderes de las comunidades, para realizar un "mapeo" de actores. Asimismo, se hizo una valoración del interés que tenían de participar en el proyecto.

c. Diagnóstico Participativo: se convocó a un taller para identificar cuáles eran los problemas más importantes que afectaban a la comunidad. En el diagnóstico surgieron los temas relativos a: manejo de residuos sólidos, escases de oportunidades laborales, delincuencia y drogadicción. 
d. Establecimiento de una agenda de trabajo: se llevó a cabo un proceso participativo para definir un plan de trabajo, priorizando aquellos temas de mayor interés. En este marco se acordó trabajar sobre el manejo de residuos sólidos, esencialmente para incentivar la prevención del Dengue.

e. Fomento a la capacidad de organización comunitaria: se implementaron varios talleres con el objetivo de fortalecer las capacidades de todos los participantes, para que pudieran asumir un papel más dinámico y activo en el plan de comunicación relativo a la prevención del Dengue. En este marco, se desarrolló el tema del reciclaje con énfasis en transformación de residuos sólidos en artículos de uso cotidiano. Adicionalmente, se trabajó el tema de la comunicación asertiva y el liderazgo.

f. Elaboración de un plan de comunicación: En este proceso participaron agentes comunitarias y representantes de las instituciones públicas relacionadas con los temas de Dengue y el manejo de residuos sólidos (Dirección Regional de Salud Pococí, Área de Salud (EBAIS), Gestión Ambiental de la Municipalidad de Pococí, Supervisión de las escuelas del circuito 5 del Ministerio de Educación Pública de Guápiles). A partir de esta articulación de esfuerzos, se realizaron cuatro sesiones para el establecimiento de acciones conjuntas, dentro de la campaña denominada "Ambiente Limpio, Comunidad Feliz".

g. Implementación de la campaña: la campaña se concentró en la eliminación de posibles criaderos del Dengue, a partir de un manejo adecuado de residuos sólidos en los hogares. Adicionalmente, y con el fin de contribuir con este propósito, se coordinó un sistema gratuito de recolección de residuos reciclables. El servicio se complementó con los talleres de "mi casa verde", que son espacios de co-aprendizaje sobre reciclaje, eliminación o transformación de criaderos y recuperación de residuos por medio de la reutilización de materiales.

h. Evaluación: con el fin de medir el alcance e impacto de la campaña, se administró una encuesta de conocimientos, actitudes y prácticas en torno a la prevención del Dengue antes y después de la implementación de la campaña.

Ficha resumen:

Proyecto: Investigación conjunta UCR / KU

Tema: Prevención del Dengue en la Zona Atlántica

Fecha de ejecución: 2011-2014

Docentes involucrados:

- Kansas University: Mugur Geana

- Universidad de Costa Rica:

- Dra. Lissette Marroquín, Escuela de Ciencias de la Comunicación Colectiva (ECCC)

- M.Sc. Adrián Avendaño

- Msc. Yanet Martínez

- M.Sc. Gabriela Murillo

- Bach. Carlos Murcia

$\circ \quad$ M.Sc. Elsy vargas

- Total de beneficiarios estimados: 125,962 habitantes del cantón de Pococí

\section{El desarrollo de cursos duales en la docencia. Caso: Curso de Comunicación y Turismo}

Uno de los principales retos que se ha definido en el marco de la internacionalización, es la posibilidad de construir, de manera coordinada y conjunta, estrategias metodológicas para la implementación 
de cursos que faculten la interacción y la construcción de aprendizajes significativos entre grupos de diversas universidades.

Para estos efectos, es importante indicar que el término "dual" adoptado no corresponde a una dualidad metodológica en respuesta al uso de elementos virtuales y presenciales (sincrónicos y/o asincrónicos), sino a la posibilidad de desarrollar dos signaturas de manera paralela, pero con puntos de coincidencia, a través de los cuales se faculta la posibilidad de compartir experiencias interculturales, elementos conceptuales y visiones profesionales, en relación con un mismo tema y/o caso de estudio.

\section{El caso del curso dual entre la Universidad de Costa Rica y la Universidad Estatal de Kansas: Comunicación y Turismo}

En el año 2014 se implementó el primer curso "piloto", bajo la modalidad de un concepto dual, entre la Universidad Estatal de Kansas (KSU) y la Universidad de Costa Rica (UCR), procurando una interacción directa entre las dos Escuelas de Comunicación, a partir del interés en un tema común: Comunicación y Turismo.

Este proceso surgió como una iniciativa en una conversación con la profesora Bárbara deSanto (KSU), Carolina Carazo y Harold Hütt (UCR), a partir de lo cual se valoró la posibilidad de lograr la implementación de esta signatura, como un curso optativo para los estudiantes de ambos centros educativos, siendo que el propósito estaría enmarcado en el desarrollo de una iniciativa conjunta.

Es así como se inicia el proceso de diseño y planificación, contemplando desde luego, una estrategia para informar a los estudiantes sobre el proyecto, siendo que para este segundo proceso, se habían definido algunos parámetros generales, tanto orientados al nivel de avance de la carrera, como a las habilidades y destrezas que debían ser requeridos en ambos grupos.

Como parte del proceso se llevó a cabo la definición de un período interciclo (dado que el curso sería intensivo para aprovechar al máximo los procesos de interacción), y adicionalmente se había previsto que implicara una movilidad docente y estudiantil hacia ambos centros educativos. Este proceso implicó varias sesiones de planificación con el fin de abordar todos los aspectos conceptuales y metodológicos del curso.

\section{Implementación del curso}

El curso dio inicio el día 7 de enero del 2014 y finalizó el día 29 de ese mismo mes, contando con la participación de los profesores nacionales Carolina Carazo y Harold Hütt, y de los profesores de la Universidad Estatal de Kansas, Bárbara DeSanto y Joye Gordon, así como también, se contó con la participación de 5 estudiantes nacionales, 10 procedentes de KSU y 7 que llevaron el curso de manera virtual desde KSU.

La metodología de trabajo fue teórico - práctica y se implementó en un horario de 8 a.m. a 5 p.m. durante las primeras dos semanas presenciales, y posteriormente se trabajó a través de sesiones virtuales. Para abordar los contenidos teóricos se contó con el apoyo magistral de los profesores, así como también, participaron conferencistas especializados, tales como: la señora Ruth Alfaro, del Área de Promoción del Instituto Costarricense de Turismo (ICT); el señor Edgar Mata, Vicepresidente de Comunicación Corporativa Ketchum para Centroamérica y el señor Gustavo Araya, Vicepresidente de la Junta Directiva del Hotel Herradura.

Asimismo, se contó con el componente práctico, dado que se trabajó, a partir de una coordinación previa con el Instituto Costarricense de Turismo (ICT), con las siguientes propiedades o proyectos turísticos:

1. Proyecto Piedra Blanca, Escazú

2. Nacientes de Palmichal, Palmichal de Acosta

3. Sueños del Bosque, San Gerardo de Dota 
La metodología implementada consistió en que varios días previos al curso, cada estudiante recibió información general sobre Costa Rica y el sector turístico, y durante la primera semana de clases - principalmente-se programaron varias giras a estos destinos, con el fin de que los estudiantes conocieran a sus propietarios, y tuvieran la oportunidad de conocer la generalidad de sus negocios y sus necesidades básicas de comunicación.

Por otra parte, se habían sostenido conversaciones previas con los propietarios de estos lugares, para facilitarles información general sobre el curso y sus objetivos, con el fin de que fueran identificando, previo a las visitas, necesidades puntuales de comunicación que los estudiantes pudieran contribuir a resolver en un corto plazo.

Asimismo, durante los dos primeros días de clases se conformaron los equipos de trabajo, compuestos por estudiantes de KSU y UCR, con el fin de promover un trabajo mixto, y de promover la interacción cultural. De esta forma, cuando se realizaron las primeras visitas, ya cada grupo sabía de previo con cual propiedad trabajaría, y durante su estancia en este, sostuvieron reuniones con los respectivos propietarios, con el objetivo de puntualizar detalles sobre los proyectos.

Desde luego, y debido al corto tiempo para trabajar en éstos, se trataba de proyectos y actividades muy puntuales tales como: propuestas para replantear la estrategia de redes sociales, diseñar y/o traducir desplegables informativos, tomar fotografías que sirvieran posteriormente para fines ilustrativos y discusión de estrategias de difusión y mercadeo, entre otros.

\section{Recomendaciones generales}

Después de haber implementado esta primera experiencia para el desarrollo de cursos duales, y con base en los elementos expuestos anteriormente, se pueden extraer las siguientes conclusiones:

a. Planificar el trabajo con al menos un año de antelación: el proceso de planificación de cursos duales implica definir los siguientes elementos: profesores involucrados, temas de interés, diseño del programa, período en el que se impartirá el curso, así como lineamientos académicos y administrativos. Adicionalmente, deberá perfeccionarse un "convenio" entre las partes y el desarrollo de actividades de promoción y reclutamiento para los estudiantes de ambos centros educativos. Es por esto que se recomienda realizar este proceso, con al menos un año de antelación, para tener la posibilidad de cumplir con cada una de estas actividades oportunamente.

b. Fortalecer los procesos de difusión para la matrícula de estudiantes extranjeros y locales: aún y cuando este elemento se menciona en el punto anterior, se hace énfasis en el proceso de difusión y reclutamiento, para que los cursos cuenten con la participación y asistencia adecuados, dado que este tipo de ofertas académicas salgan de la cotidianidad, lo que implica convencer, de alguna manera, a los estudiantes para que se interesen en asumir este tipo de retos, y puedan visualizar los beneficios que en el corto, mediano y largo plazo, este tipo de esfuerzos les pueden generar.

c. Promover mayor interactividad en actividades sociales y culturales durante el período de interacción de los grupos: uno de los elementos que más rescataron los estudiantes, fue lo agradable y positivo que resultó para ellos tener espacios de interacción al margen del curso, los cuales en el este caso en particular fueron relativamente escasos, por lo que se ha hecho hincapié para que en futuras ocasiones, se incentive una agenda paralela de orden social, que facilite la interacción entre ambos grupos, de una forma más espontánea, para propiciar y enriquecer el intercambio cultural.

d. Fortalecer la plataforma virtual para incluir en esta lecturas, las prácticas y rúbricas de los proyectos: siendo que no todos los estudiantes tendrán la oportunidad de participar de manera presencial en este tipo de cursos, es de gran importancia contar con una plataforma virtual que facilite la interacción remota, así como el suministro de materiales didácticos y el trabajo colaborativo. Dentro de las posibilidades para estos efectos pueden figurar las plataformas Moodle, Blackboard, PB Workds, Google Hangouts, entre otras. 
e. Mantener el esquema de prácticas duales, bajo el esquema teórico - práctico: los estudiantes enfatizaron los beneficios derivados de los ejercicios prácticos, producto de los cuales pudieron desarrollar proyectos para "clientes reales", como lo manifestaron algunos de ellos en sus hojas de evaluación, lo cual les motivó a poner en práctica el conocimiento adquirido en el curso, y desde luego, las habilidades, destrezas y conceptos adquiridos a través de sus respectivos planes de estudios. Desde luego, esto tuvo un elemento adicional de gran importancia, y fue la posibilidad de incentivar la discusión de estrategias y el intercambio de ideas y propuestas para encontrar las mejores soluciones a las necesidades de los clientes, en con el valor agregado de requerir la adaptación de éstas a contextos ajenos.

f. Dar continuidad a la toma de fotografías durante el proceso y compartir los comentarios en el Facebook de internacionalización para que se compartan algunas de las experiencias vividas: la toma de fotografías, y en algunos casos de videos, fue muy producente para la generación de material ilustrativo para los reportes diarios que debían elaborar los participantes, en los cuales exponían sus principales lecciones de aprendizaje, sino también, para ilustrar los informes de los proyectos, y más importante aún, para facilitar material a los propietarios de los proyectos, que pueda ser utilizado en las estrategias propuestas, o bien, en futuras actividades de divulgación. Por otra parte, lograr que se compartieran comentarios y fotografías en el Facebook fue de gran valía, dado que otros estudiantes pudieron darse una idea de las situaciones, prácticas y aprendizajes experimentados por sus compañeros, así como también, según han manifestado de manera informal a lo interno de la ECCC, se han sentido motivados para participar en futuras experiencias.

g. Procurar, por parte de los docentes, sistematizar las actividades desarrolladas con el fin de generar antecedentes que sirvan de referencia para futuros docentes: documentar las experiencias en el uso de metodologías didácticas utilizadas en proyectos colaborativos y cursos duales será de gran importancia para facilitar el desarrollo de proyectos similares por parte de otros docentes. Procurar que nuevos docentes se involucren en iniciativas de este tipo: es determinante procurar la participación de más docentes en este tipo de proyectos, para tener la oportunidad de establecer más vínculos de cooperación, en diversos temas de interés, generando mayores oportunidades de aprendizaje y crecimiento para los estudiantes. Desde luego, esto implica asumir un reto y afrontar nuevos escenarios, tales como la planificación didáctica conjunta con las contrapartes, el diseño de estrategias metodológicas y creativas, y la motivación de los estudiantes para participar de estas oportunidades.

h. Propiciar la creación de un fondo de apoyo a estudiantes locales para que puedan vivir este tipo de experiencias en otros países: una de las metas que está pendiente de concretarse en la Estrategia de Internacionalización, es la movilidad estudiantil, dado que los pronósticos para lograr la obtención de fondos que permitan patrocinar el viaje de nuestros estudiantes a otras universidades ha sido limitado. No obstante, si logramos desarrollar este tipo de esfuerzos de manera sistemática, los costos de matrícula de los estudiantes extranjeros, permitirán ir generando un fondo interno para la cobertura de los gastos de traslado, hospedaje y alimentación de nuestros alumnos, y generar así, una experiencia vivencial de mayor significancia y profundidad para ellos.

i. Validar y analizar las metodologías de aprendizaje y las dinámicas implementadas, con el fin de realizar un proceso de evaluación constante y mejoramiento continuo: además de realizar la sistematización de esfuerzos y experiencias, es de vital importancia realizar procesos de análisis y valoración, con el fin de ir identificando prácticas idóneas para estimular el aprendizaje colaborativo, y de propiciar una mayor motivación e interacción entre las partes.

j. Difundir por diversas vías las acciones ejecutadas: a través de publicaciones, conferencias, reuniones y talleres, entre otros, con el fin de compartir las experiencias aprendidas con otros docentes y escuelas que puedan tener interés en participar en este tipo de iniciativas. 
k. Es esencialmente importante dar continuidad y fortalecer la estrategia de internacionalización de la ECCC, con el fin de procurar más y mejores oportunidades para docentes y estudiantes: esto para mejorar sus perfiles de salida, impulsar el manejo de otros idiomas y procurar innovaciones metodológicas, así como el uso de tecnologías de la información de vanguardia. Para esto es determinante el apoyo de las autoridades de la Escuela y de la Universidad, tanto en la emisión de lineamientos y directrices que respalden las acciones ejecutadas y propuestas, así como también, contribuir con el suministro de recursos. Sin lugar a dudas, este proceso contribuirá en gran medida a formar mejores profesionales, mejores personas, y a lograr una mejor comprensión del mundo globalizado, las necesidades y características de los diversos contextos, así como también, facilitará dotar a los participantes de mejores herramientas para enfrentar los retos actuales y futuros, en un mercado tan dinámico y cambiante.

\section{Ficha resumen:}

Proyecto: Curso dual entre la Universidad de Costa Rica y la Universidad Estatal de Kansas

Tema: Comunicación y Turismo

Fecha de ejecución: Interciclo 2014

Docentes involucrados:

- Kansas State University:

- Bárbara deSanto

- Joye Gordon

- Universidad de Costa Rica:

- Carolina Carazo

- Harold Hütt

Total de beneficiarios: 17 estudiantes (5 nacionales) y 3 empresas de turismo rural comunitario.

\section{Conclusiones Generales}

a. Los proyectos y actividades de internacionalización permiten motivar a docentes y estudiantes a intercambiar criterios y experiencias, así como también a resolver problemas de interés común, o bien, de una localidad en particular a partir de una construcción conjunta.

b. La tecnología brinda múltiples alternativas para facilitar este tipo de procesos e interacciones, donde únicamente se requiere de la participación activa del personal docente en la planificación e implementación de este tipo de proyectos.

c. La multiculturalidad es un factor de gran importancia para las partes, dado que permite una comprensión de un entorno, cada vez más globalizado.

d. Las interacciones internacionales permiten reafirmar en los estudiantes el conocimiento adquirido y permiten motivar discusiones y análisis con múltiples puntos de vista, en el marco de contextos distintos.

e. Este tipo de proyectos deben ser desarrollados de manera sistemática en la carrera, para impulsar un esquema lo más inclusivo posible, que permita abordar diversos contenidos y casos, con diversas contrapartes en distintas localidades y contextos, lo que implicará un enriquecimiento cultural y académico de los participantes. 
f. Es necesario impulsar la internacionalización desde la docencia, la acción social y la investigación, con el fin de lograr un mayor impacto y transversalidad en su aplicación.

\section{Referencias}

Aguilar, Yorleni y Riveros, Alba (2013). Tesis de Licenciatura: Evolución del modelo de internacionalización y enfoque regional de la Universidad de Costa Rica. San José, Costa Rica.

Barbero, Alberto (2011). Dinámicas participativas: Phillips 66. Recuperado de http://www.albarbero. com/2011/01/phillips-66.html

Benavidez, Jorge (2001). El acceso al desarrollo pedagógico en el nuevo contexto de la tecnología de la información en educación. Revista Meridiano. Vol 36 / Facultad de Educación, Universidad de Nariño.

Carazo, Carolina y Hütt, Harold (2011). Estrategia de Internacionalización. Escuela de Ciencias de la Comunicación Colectiva.

Consejo Nacional de Rectores (2005). Plan Nacional de la Educación Superior Universitaria Estatal 2006-2010 (Planes 2006-2010). San José: CONARE. Oficina de Planificación de la Educación Superior, Comisión de Directores de Planificación.

Díaz Frida y Hernández Gerardo (2002). Estrategias docentes para un aprendizaje significativo. Una interpretación constructivista. Segunda Edición. México: Editorial McGraw Hill.

Didou Aupetit, S. (2014) La UNESCO y la educación superior, 2014-2017: aportes de la Reunión de Cátedras UNESCO sobre la educación superior, las TIC en la educación superior y los profesores. Recuperado de: http://www.unesco.org/fileadmin/MULTIMEDIA/HQ/ED/pdf/UNESCOsummary-report-chairs-2014-1.pdf

Dominguez Menéndez, Jorge (s.f.) La educación superior en América Latina frente a la globalización: internacionalización o transnacionalización. Revista Cubana de Educación Superior.

Fainholc, Beatriz (1998) Nuevas Tecnologías de la Información y de la Comunicación en la Enseñanza. Buenos Aires: AIQUE.

Carmona, Juan José y Ibañez, Luis (2011). Pedagogía crítica y Web 2.0: formación del profesorado para transformar el aula. Revista Electrónica Interuniversitaria de Formación del Profesorado, Vol. 14, número 2, 81-95. Recuperado de http://www.aufop.com/aufop/uploaded_files/articulos/1311954508.pdf

Ferreiro, Ramón y DeNapoli, Anthony (2006). Un concepto clave para aplicar exitosamente las tecnologías de la educación: los nuevos ambientes de aprendizaje. Revista Panamericana de Pedagogía: saberes y quehaceres del Pedagogo, Vol. 8, 121-154.

Gacel, Jocelyne (1999) Internacionalización de la Educación Superior en América Latina y el Caribe. México.

Islas, Claudia y Martínez, Evelio (2008). El uso de las TIC como apoyo a las actividades docentes. Revista RED, setiembre 2008. Recuperado de http://www.eveliux.com/mx/El-uso-de-las-TICcomo-apoyo-a-las-actividades-docentes.html

Knight, Jane (1994). Internatioanalization: Elements and checkpoints. CBIE Research, Canadiean, Bureau of International Education No.7, 1994 (pp. 1-14).

López, Alexander (2001). Artículo de Revista. Vol 16. Introducción de las nuevas tecnologías de la información y la comunicación (TICs) en la educación. Perspectivas. Experiencias de la digitalización, ejemplos, valoración. INFOLAC: boletín trimestral del Programa Regional para el Fortalecimiento de la Cooperación entre Redes y Sistemas Nacionales de Información para América Latina y el Caribe.

Moncada Cerón, Jesús Salvador (2011). Hacia una internacionalización solidaria de la educación superior. Revista Ciencia, Arte y Humanidades Vol 20. Universidad del Bio-bio. 
Pedregal, Rafael (2003) Tesis de Maestría: La internacionalización de la educación superior en América del Norte ante los retos del TLCAN: un estudio comparativo. México. Recuperado de http:// catarina.udlap.mx/u_dl_a/tales/documentos/mes/pedregal_c_r/

Peña, Carmen Helena y Jiménez, Myriam (2014) Internacionalización y cooperación universitaria: el caso de la cátedra Europa. Revista Investigación y Desarrollo. Vol. 22. Colombia: Fundación Universidad del Norte.

Pérez, Héctor (1995). La pedagogía en el proceso educativo. Publicaciones Pedagógicas. San José, Costa Rica.

Pimienta, Julio (2008). Constructivismo. Estrategias para aprender a aprender. Tercera Edición. México: Editorial Leticia Gaona Figueroa.

Qiang, Zha (2003). Internationalization of Higher Education: towards a conceptual framework. Policity Futures in Education. Volume 1, Number 2. Recuperado de http://www.unal.edu.co/ori/redorion/docs/Conceptual-Framework-Internationalization.pdf

Rama Vitale, Claudio (2007). La despresencialización de la educación superior en América Latina: ¿tema de calidad de cobertura, de internacionalización o de financiamiento?. Revista de Innovación, Año7, número 7. México: Universidad de Guadalajara.

Ruiz, Ángel (2000) La Educación Superior en Costa Rica: Tendencias y retos en un nuevo escenario histórico. [Versión digital] Recuperado de http://issuu.com/moskar/docs/ educacion_superior_en_costa_rica\#tembed

Sáez, José; Lorraine, Jackson y Miyata, Yoshiro (2013). Uso de edmodo en proyectos colaborativos internacionales en educación primaria. Revista Electrónica de Tecnología Educativa (EDUTEC). Recuperado de http://portal.uned.es/pls/portal/docs/PAGE/UNED_MAIN/LAUNIVERSIDAD/UBICACIONES/03/DOCENTE/JOSE_MANUEL_SAEZ_LOPEZ/16EDMODO_ PROY_COLABORATIVOS_EDUTEC-E_N43-SAEZ_LORRAINE_YOSHIRO.PDF

Samper, Julián (2001) De la Escuela nueva al constructivismo. Un análisis crítico. Colombia: Editorial Normos S.A.

Serrano, María de los Ángeles (2010). Diseño de Proyectos Colaborativos. Revista e-Formadores. Recuperado de http://red.ilce.edu.mx/sitios/revista/e_formadores_pri_10/articulos/angeles_serrano_feb2010.pdf

Universidad Autónoma de México (2004) Cuadernos americanos, núm. 105. México: Editorial Universidad Autónoma de México. 
